\title{
Author Correction: Short-lived climate pollutant mitigation and the Sustainable Development Goals
}

Andy Haines (D), Markus Amann, Nathan Borgford-Parnell, Sunday Leonard, Johan Kuylenstierna and Drew Shindell (D) Correction to: Nature Climate Change https://doi.org/10.1038/s41558-017-0012-x, published online 30 November 2017.

In the version of this Perspective originally published, Fig. 1 incorrectly had two entries of 'Reduced rate of sea-level rise by $20 \%$ by 2050 '; the first entry (row 2, column 3) should instead have read 'Reduced disruption of weather patterns'. This has now been corrected in the online versions of the Perspective.

\section{Author Correction: Biomass-based negative emissions difficult to reconcile with planetary boundaries}

Vera Heck (D), Dieter Gerten, Wolfgang Lucht and Alexander Popp

Correction to: Nature Climate Change https://doi.org/10.1038/s41558-017-0064-y, published online 22 January 2018.

In the version of this Letter originally published, in Fig. 2, the labels for the yellow and green areas were swapped: the yellow areas should have been labelled 'Global uncertainty zones' and the green areas should have been labelled 'Global safe zones'. This has now been corrected in the online versions of the Letter.

\section{Publisher Correction: Recently amplified arctic warming has contributed to a continual global warming trend}

Jianbin Huang, Xiangdong Zhang (1), Qiyi Zhang, Yanluan Lin, Mingju Hao, Yong Luo (1), Zongci Zhao, Yao Yao, Xin Chen, Lei Wang, Suping Nie, Yizhou Yin, Ying Xu and Jiansong Zhang

Correction to: Nature Climate Change https:/doi.org/10.1038/s41558-017-0009-5, published online 20 November 2017.

In the version of this Letter originally published, the increments on the $y$ axis of Fig. 3 were incorrectly labelled as ' $0.0 ; 0.2 ; 0.2 ; 0.3$ '; they should have read ' $0.0 ; 0.1 ; 0.2 ; 0.3$ '. This has now been corrected in all versions of the Letter. 